\title{
Ionic Liquid Used for Charge Compensation for High-Resolution Imaging and Analysis in the FE-SEM
}

\author{
Nicolas Brodusch ${ }^{1}$, Hendrix Demers ${ }^{1}$ and Raynald Gauvin ${ }^{1}$ \\ 1. Department of Mining and Materials Engineering, McGill University, Montreal, Quebec, Canada.
}

Charging effects are inevitable when an electron beam interacts with a partially conductive target material. The conduction and valence bands being overlapped in metals, the evacuation of excess negative charges is complete whereas a gap between these two bands exists in semi-conductors and insulators, preventing an efficient electrical conductivity. Thus, the excess negative charges brought by the electron beam stay trapped at the shallow surface of the specimen and generates a negative electric field at the surface while a positive field is created deep inside the interaction volume [1]. To help reducing these effects, coating with a conductive material [2], determining the neutral accelerating voltage [3] or using positively charged ions in the vicinity of the impact point of the electron beam [4] may generally be used. However, the coating technique, which is the most used, results in a loss of topographic and diffraction contrast in the images.

Since the last ten years, a great interest has been focused on ionic liquids (ILs) in high vacuum because they provide a very low vapor pressure and high conductivity while staying in liquid state. Hence, their use has been increasing in the scanning electron microscopy (SEM) field, especially for biological [5] and ceramic [6] applications. Here, we demonstrate the usefulness of 1-butyl-3-methylimidazolium tetrafluoroborate $\left(\mathrm{BMI}_{-} \mathrm{BF}_{4}\right)$ for reducing charging in mining ores and lithium-ion batteries electrode nanomaterials. In the former, the high mixing of different minerals generates a different level of charging requiring carbon coating. However, the coating absorbs a high amount of diffracted backscattered electrons (BSEs) preventing from acquiring electron backscatter diffraction (EBSD) orientation maps, while the material still exhibit charging deep below the surface. The immersion of the mineral block in a diluted solution of $\mathrm{BMI}_{-} \mathrm{BF}_{4}$ in ethanol provided more electrical stability enabling the acquisition of long EBSD maps with high resolution (Figure 1). This permitted to improve the knowledge of the fergusonite containing clusters for the improvement of the extraction process [7].

The $\mathrm{Li}_{2} \mathrm{MSiO}_{4}$ nanomaterials ( $\mathrm{M}$ being a transition metal) used in the last generation of lithium-ion batteries electrodes are highly non-conductive due to their silicate intrinsic nature and imaging and chemical mapping are disturbed by charging effects. However, when the powder is mixed with a diluted

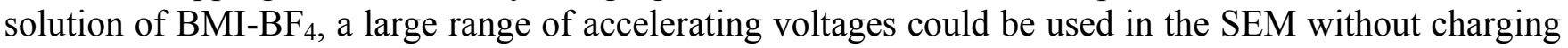
allowing secondary (SE) and BSE imaging with high spatial resolution (Figure 2).

This work, completing the results from previous studies on the subject, shows how ILs can help in the characterization of non-conductive minerals and nanoparticles with no or low amount of charging.

\section{References:}

[1] J. Cazaux, Microscopy and Microanalysis 10 (06) (2004), p. 670-684.

[2] P. Echlin (ed.) in "Handbook of sample preparation for scanning electron microscopy and x-ray microanalysis", (Springer, New York) (2009), 330p.

[3] DC. Joy, C. Joy, Microscopy and Microanalysis 4 (1998), p. 475-480.

[4] K. Robertson, R. Gauvin and J. Finch, Microscopy and Microanalysis 10 (2004), p. 721-732.

[5] Y. Ishigaki et al. Microscopy Research and Technique 74 (5) (2011), p. 415-420. 
[6] C. Takahashi et al, Ceramics International 39 (2) (2013), p. 1065-1073. [7] N. Brodusch et al, Microscopy Research and Technique (2014).

(a)

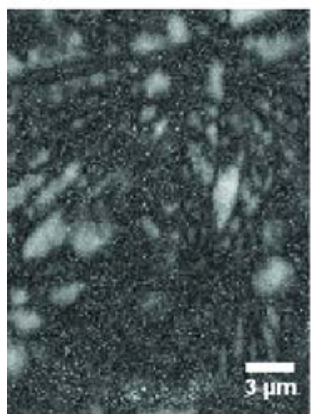

(d)

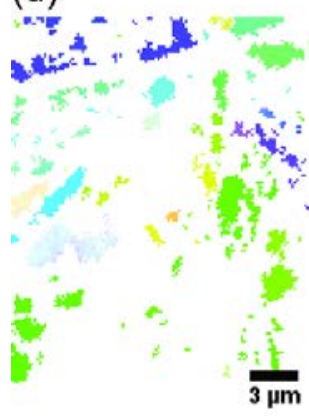

(b)

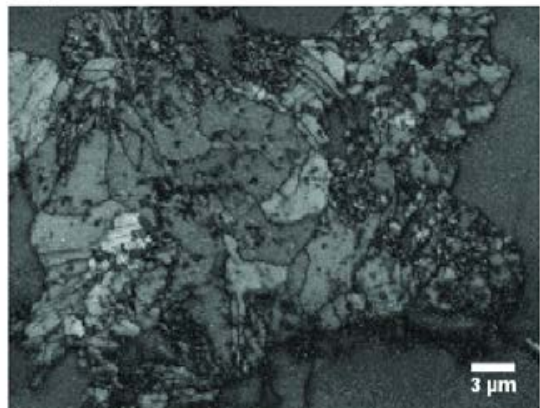

(e)

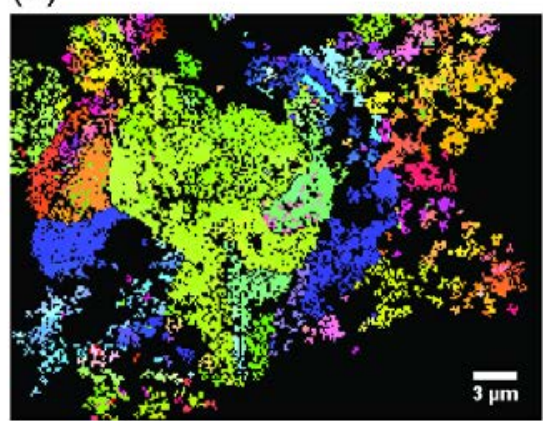

(c)

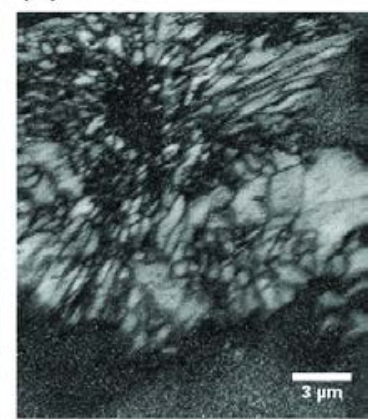

(f)

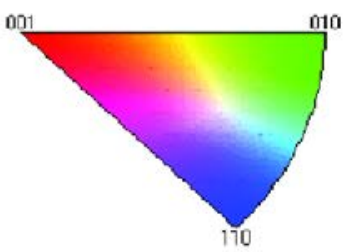

Figure 1. Electron backscatter diffraction maps of a fergusonite/zircon cluster in the Nechalacho ore at $20 \mathrm{kV}$. (a, b, c) Band contrast maps, (d, e) Inverse pole figure (IPF) maps corresponding to (a) and (b) respectively. (f) IPF legend. (a), (c) from carbon coated sample, (b) from ionic liquid treated sample.
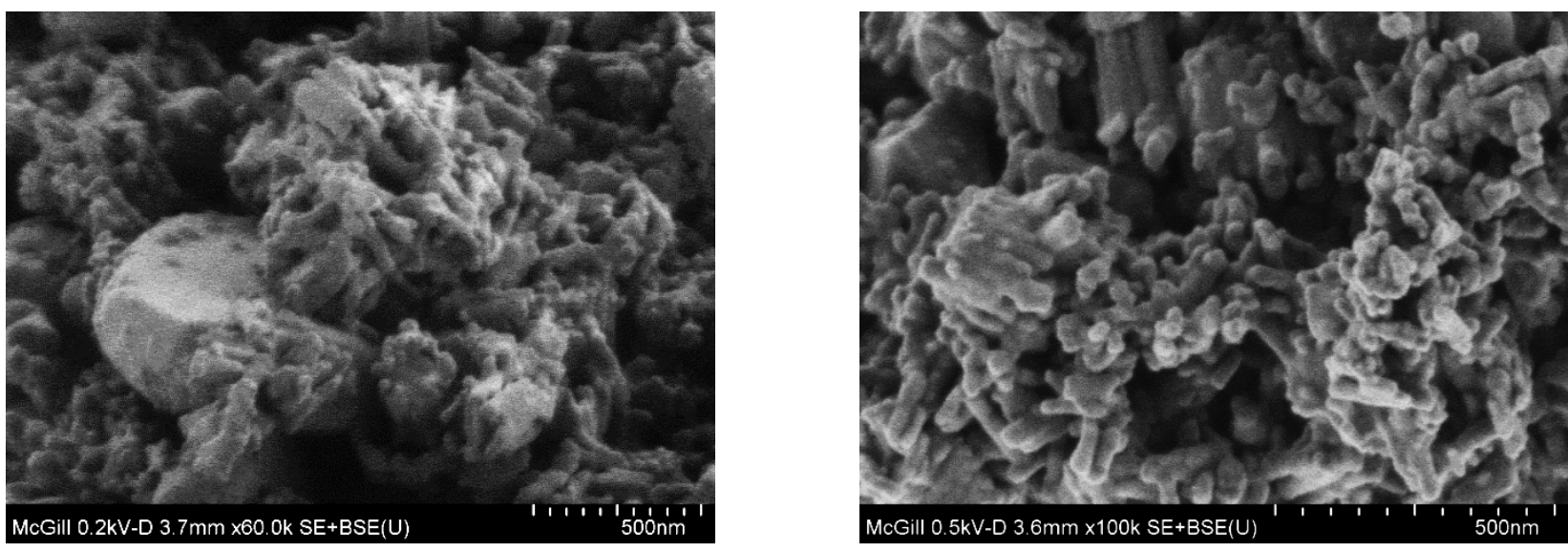

Figure 2. Low accelerating voltage secondary and backscatter electron (SE+BSE) images of $\mathrm{Li}_{2} \mathrm{MnSiO}_{4}$

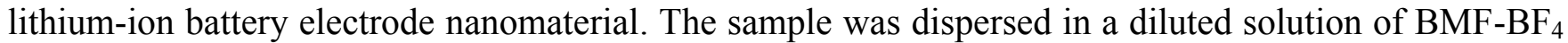
with ethanol prior to observation. 